\title{
Europäischer Tarifbericht des WSI 2009/2010
}

\begin{abstract}
Mit einiger Zeitverzögerung hat dieaktuelle Weltwirtschaftkrise auch dieEntwicklung der Löhne erreicht. Während die Tariflohnabschlüsse 2009 in Europa noch relativ hoch waren, dürften sie 2010 deutlich niedriger ausfallen. Bei den Effektivlöhnen lässt sich bereits für 2009 eine stark rückläufige Entwicklungstendenz beobachten, die sich 2010 zu verstärken droht. In vielen europäischen Ländern fordern Arbeitgeber und Regierungen mittlerweile, die Löhne einzufrieren, - oder sie sprechen sich sogar offen für Lohnkürzungen aus. Damit aber würde die Lohnpolitik - anstatt über angemessene Lohnerhöhungen den privaten Konsum zu stärken - dazu beitragen, dass sich die Krise weiter verlängert.
\end{abstract}

\section{1 \\ Ökonomische Rahmenbe- dingungen der Tarifpolitik}

\subsection{ALLGEMEINE WIRTSCHAFTS- ENTWICKLUNG}

Die Tarifauseinandersetzungen des Jahres 2009 standen ganz im Vorzeichen der schwersten und tiefsten Wirtschaftskrise in der Geschichte der Europäischen Union (EU), die in den meisten europäischen Ländern zu einem dramatischen Rückgang der Wirtschaftleistung geführt hat. Innerhalb der EU 27 ist das Bruttoinlandsprodukt (BIP) insgesamt um 4,1\% gegenüber dem Vorjahr zurückgegangen (Tabelle 1). ${ }^{1}$

Den stärksten Wachstumseinbruch mussten die baltischen Staaten hinnehmen, deren BIP sich um knapp $14 \%$ in Estland und um $18 \%$ in Lettland und Litauen verminderte. Innerhalb der alten EU-Staaten erlebte Irland mit minus $7,5 \%$ den stärksten Wirtschaftsrückgang, gefolgt von Finnland mit minus 6,9\% und Deutschland mit minus $5 \%$. Der Rückgang der Wirtschaftsleistung war insgesamt in denjenigen Ländern besonders ausgeprägt, die eine hohe Exportabhängigkeit ausweisen und dementsprechend besonders stark unter dem Einbruch des Welthandels litten. Demgegenüber war z. B. das stärker binnenwirtschaftlich orientierte Frankreich mit einem rückläufigen BIP von minus 2,2\% deutlich geringer von der Krise betroffen. Polen konnte sogar als einziges EU-Land trotz Weltwirtschaftskrise mit einer Steigerung des BIP um 1,2\% eine positive Wachstumsrate verzeichnen.
Für das Jahr 2010 geht die Europäische Kommission davon aus, dass die Talsohle der Wirtschaftkrise bereits durchschritten sei und es wieder zu einer leichten Erholung der Konjunkturentwicklung komme. Insgesamt prognostiziert sie für die 27 EU-Staaten ein BIP-Wachstum von 0,7 \%, wobei die Spannbreite von minus $4 \%$ in Lettland und Litauen bis zu plus $2 \%$ in Polen und der Slowakei reicht. Allerdings betont die Europäische Kommission, dass ihre Prognose mit einem hohen Maß an Unsicherheit verhaftet sei, die vor allem aus der nach wie vor instabilen Entwicklung der internationalen Finanzmärkte resultiert (Europäische Kommission 2010a).

\subsection{DIE LAGE AUF DEM ARBEITSMARKT}

Angesicht der tiefen Wirtschaftskrise hat sich auch die Lage auf dem Arbeitsmarkt in den meisten EU-Staaten deutlich verschlechtert. Im Jahr 2009 gab es offiziell innerhalb der EU 27 etwa 21,5 Mio. Arbeitslose, was nach der Definition von Eurostat einer Arbeitslosenquote von 8,9 \% entspricht (Europäische Kommission 2010b). Gegenüber dem Vorjahr hat sich die Anzahl der Arbeitslosen damit um mehr als 4,5 Mio. vergrößert.

Das Ausmaß, in dem die Arbeitslosigkeit in den einzelnen EU-Staaten zugenommen hat, weist jedoch erhebliche Unterschiede auf. Mit einem Zuwachs von mehr als neun Prozentpunkten stieg die Arbeitslosenquote 2009 in den baltischen Staaten am stärksten und lag im Jahresdurchschnitt bei $14 \%$ (Estland und Litauen) bzw. 17,6 \% (Lettland). Zu einem besonders hohen Anstieg der Ar- beitslosigkeit kam es auch in Spanien, wo die Arbeitslosenquote von 11,3 \% (2008) auf $18,1 \%$ (2009) zunahm und damit den EU-weit höchsten Wert aufweist. Ebenfalls stark angestiegen ist die Arbeitslosenquote im krisengeschüttelten Irland, wo sie sich innerhalb eines Jahres von 6,0 \% (2008) auf $11,8 \%$ (2009) fast verdoppelt hat.

Demgegenüber gibt es auch eine Reihe von Ländern, wo trotz eines starken Wirtschaftseinbruchs die Arbeitslosigkeit nur sehr moderat angestiegen ist. Hierzu gehören vor allem Deutschland, Belgien und die Niederlande, wo der Zuwachs der Arbeitslosenquote sich unterhalb eines Prozentpunktes bewegte. In diesen Ländern hat insbesondere die Nutzung von Kurzarbeit und anderen Maßnahmen der Arbeitszeitverkürzung einen stärkeren Anstieg der Arbeitslosenzahlen verhindert (European Foundation 2009).

Anders als bei der Entwicklung des Wirtschaftwachstums geht die Europäische Kommission bei der Entwicklung auf dem Arbeitsmarkt davon aus, dass der Wendepunkt noch keinesfalls erreicht ist.

\footnotetext{
1 Im Folgenden wird, falls nicht anders ausgewiesen, auf Daten des EU-Statistikamtes Eurostat und der AMECO-Datenbank der Europäischen Kommission zurückgegriffen. Bei den Angaben für 2009 handelt es sich teilweise noch um vorläufige Daten. Bei den Angaben für 2010 handelt es sich um Prognosedaten der Europäischen Kommission (Europäische Kommission 2009, 2010a).
}

Thorsten Schulten, Dr. Wissenschaftler im WSI in der Hans-Böckler-Stiftung. Arbeitsschwerpunkte: Arbeits- und Tarifpolitik in Europa. e-mail: Thorsten-Schulten@boeckler.de 
Tabelle 1: Wachstum und Arbeitslosigkeit in Europa 2007-2010

\begin{tabular}{|c|c|c|c|c|c|c|c|c|}
\hline & \multicolumn{4}{|c|}{ Bruttoinlandsprodukt } & \multicolumn{4}{|c|}{ Arbeitslosigkeit } \\
\hline & 2007 & 2008 & 2009 & 2010 & 2007 & 2008 & 2009 & 2010 \\
\hline \multicolumn{9}{|l|}{ Alte EU-Staaten } \\
\hline Belgien & 2,9 & 1,0 & $-2,9$ & 0,6 & 7,5 & 7,0 & 7,9 & 9,9 \\
\hline Dänemark & 1,6 & $-1,2$ & $-4,5$ & 1,5 & 3,8 & 3,3 & 6,0 & 5,8 \\
\hline Deutschland & 2,5 & 1,3 & $-5,0$ & 1,2 & 8,4 & 7,3 & 7,7 & 9,2 \\
\hline Finnland & 4,2 & 1,0 & $-6,9$ & 0,9 & 6,9 & 6,4 & 8,2 & 10,2 \\
\hline Frankreich & 2,3 & 0,4 & $-2,2$ & 1,2 & 8,4 & 7,8 & 9,4 & 10,2 \\
\hline Griechenland & 4,5 & 2,0 & $-1,1$ & $-0,3$ & 8,3 & 7,7 & 9,0 & 10,2 \\
\hline Großbritannien & 2,6 & 0,5 & $-4,8$ & 0,9 & 5,3 & 5,6 & 7,8 & 8,7 \\
\hline Irland & 6,0 & $-3,0$ & $-7,5$ & $-1,4$ & 4,6 & 6,0 & 11,8 & 14,0 \\
\hline Italien & 1,6 & $-1,0$ & $-4,7$ & 0,7 & 6,1 & 6,7 & 7,8 & 8,7 \\
\hline Luxemburg & 6,5 & 0,0 & $-3,6$ & 1,1 & 4,2 & 4,9 & 5,7 & 7,3 \\
\hline Niederlande & 3,6 & 2,0 & $-4,5$ & 0,3 & 3,2 & 2,8 & 3,5 & 5,4 \\
\hline Österreich & 3,5 & 2,0 & $-3,7$ & 1,1 & 4,4 & 3,8 & 5,0 & 6,0 \\
\hline Portugal & 1,9 & 0,0 & $-2,9$ & 0,3 & 8,1 & 7,7 & 9,6 & 9,0 \\
\hline Schweden & 2,6 & $-0,2$ & $-4,6$ & 1,4 & 6,1 & 6,2 & 8,3 & 10,2 \\
\hline Spanien & 3,6 & 0,9 & $-3,6$ & $-0,8$ & 8,3 & 11,3 & 18,1 & 20,0 \\
\hline \multicolumn{9}{|l|}{ Neue EU-Staaten } \\
\hline Bulgarien & 6,2 & 6,0 & $-5,9$ & $-1,1$ & 6,9 & 5,6 & 6,7 & 8,0 \\
\hline Estland & 7,2 & $-3,6$ & $-13,7$ & $-0,1$ & 4,7 & 5,5 & 14,0 & 15,2 \\
\hline Lettland & 10,0 & $-4,6$ & $-18,0$ & $-4,0$ & 6,0 & 7,5 & 17,6 & 19,9 \\
\hline Litauen & 9,8 & 2,8 & $-18,1$ & $-3,9$ & 4,3 & 5,8 & 14,0 & 17,6 \\
\hline Malta & 3,7 & 2,1 & $-2,2$ & 0,7 & 6,4 & 5,9 & 7,0 & 7,4 \\
\hline Polen & 6,8 & 5,0 & 1,2 & 1,8 & 9,6 & 7,1 & 8,2 & 9,9 \\
\hline Rumänien & 6,3 & 6,2 & $-8,0$ & 0,5 & 6,4 & 5,8 & 9,0 & 8,7 \\
\hline Slowakei & 10,4 & 6,4 & $-5,8$ & 1,9 & 11,1 & 9,5 & 11,9 & 12,8 \\
\hline Slowenien & 6,8 & 3,5 & $-7,4$ & 1,3 & 4,9 & 4,4 & 6,0 & 8,3 \\
\hline Ungarn & 1,0 & 0,6 & $-6,5$ & $-0,5$ & 7,4 & 7,8 & 10,0 & 11,3 \\
\hline Tschechien & 6,1 & 2,5 & $-4,8$ & 0,8 & 5,3 & 4,4 & 6,8 & 7,9 \\
\hline Zypern & 4,4 & 3,7 & $-0,7$ & 0,1 & 4,0 & 3,6 & 5,3 & 6,6 \\
\hline Gesamte EU 27 & 2,9 & 0,8 & $-4,1$ & 0,7 & 7,1 & 7,0 & 8,9 & 10,3 \\
\hline Norwegen & 3,1 & 2,1 & $-1,5$ & 0,6 & 2,5 & 2,5 & 4,8 & 5,0 \\
\hline Schweiz & 3,6 & 1,8 & $-2,4$ & $-0,1$ & 3,4 & 3,2 & 4,8 & 6,4 \\
\hline \multicolumn{9}{|c|}{$\begin{array}{l}\text { Bruttoinlandsprodukt }=\text { Veränderung des BIP in \% gegenüber dem Vorjahr } \\
\text { Arbeitslosenquote }=\text { Anzahl der Arbeitslosen in \% der zivilen Erwerbsbevölkerung (Eurostat-Definition) } \\
\text { Angaben für 2009: Teilweise vorläufige Daten } \\
\text { Angaben für 2010: Prognose der Europäischen Kommission }\end{array}$} \\
\hline Quelle: Eurostat. & & & & & & & IITTE & NGEN \\
\hline
\end{tabular}

Für das Jahr 2010 prognostiziert sie insgesamt einen weiteren Anstieg der Arbeitslosenzahlen um zusätzlich 4,5 Mio. Damit wären EU-weit knapp 25 Mio. Personen arbeitslos, was einer Arbeitslosenquote von 10,3\% entspricht. Mit Ausnahme von Dänemark und Portugal wird für alle EUStaaten eine weitere Zunahme der Arbeitslosigkeit vorhergesagt. Für Deutschland erwartet die Europäische Kommission einen Anstieg der Arbeitslosenquote von $7,7 \%$ auf $9,2 \%$.

\subsection{PREIS- UND PRODUKTIVITÄTS- ENTWICKLUNG}

Die Tarifauseinandersetzungen in Europa orientieren sich traditionell neben der Konjunkturentwicklung und der Lage auf dem Arbeitsmarkt vor allem an der Entwicklung der Preise und der Arbeitsproduktivität (Tabelle 2). Die Summe beider
Indikatoren bildet den lohnpolitischen Verteilungsspielraum, dessen Ausschöpfung durch entsprechende Lohnerhöhungen den Beschäftigten eine gleichgewichtige Partizipation an der allgemeinen Wirtschaftentwicklung garantiert und das Verhältnis zwischen Arbeits- und Kapitaleinkommen konstant hält. Makroökonomisch gilt die produktivitätsorientierte (Real-)Lohnpolitik als wachstums- und stabilitätsgerecht, da sie die private Konsumnachfrage fördert, ohne die Geldwertstabilität zu gefährden. Bereits seit Ende der 1990er Jahre haben sich die europäischen Gewerkschaften als Zielmarke für eine europäische Koordinierung der Tarifpolitik auf eine produktivitätsorientierte (Real-)Lohnpolitik verständigt, die die nationalen Verteilungsspielräume ausschöpft, um auf diese Weise gegenseitiges Lohndumping zu verhindern (Schulten 2009).
Bei der Definition des tarifpolitischen Verteilungsspielraumes als Summe aus Preis- und Produktivitätsentwicklung ist in der Wirtschaftswissenschaft strittig, welche konkreten Indikatoren insbesondere für die Preisentwicklung verwendet werden sollen. Gegenüber möglichen alternativen Indikatoren (wie z. B. der Kerninflationsrate, der EZB-Zielinflationsrate oder der Entwicklung der Erzeugerpreise) wird im Folgenden bei der Bestimmung der nationalen Verteilungsspielräume die jeweils nationale Entwicklung der Verbraucherpreise zugrunde gelegt. Aus Arbeitnehmersicht stellen diese den wichtigsten ökonomischen Indikator dar, weil sie über das reale Lohnniveau und die damit verbundene Kaufkraft entscheiden. Dementsprechend bilden die nationalen Verbraucherpreise für die Gewerkschaften die bedeutsamste Orientierungsgröße bei den Tarifauseinandersetzungen.

Das Jahr 2009 weist insgesamt eine äußerst schwache Preisentwicklung auf, was zum einen an dem Rückgang der Rohstoff- und Energiepreise, zum andern an der allgemeinen Schwäche der gesamtwirtschaftlichen Nachfrage in der Krise liegt. Nachdem der Harmonisierte Verbraucherpreisindex (HVPI) 2008 im EUDurchschnitt noch um 3,7 \% angestiegen war, lag die Zuwachsrate 2009 nur noch bei $1 \%$ und damit deutlich unterhalb der Zielinflationsrate der EZB von $2 \%$ (Tabelle 2). In einigen Ländern wie Portugal, Spanien oder der Schweiz kam es sogar zu einer leicht negativen Preisentwicklung. In Irland ging das Preisniveau sogar um 1,7 \% zurück und nahm damit einen offen deflationären Verlauf. In den meisten EU-Staaten lag der Anstieg der Verbraucherpreise zwischen $0 \%$ und $1 \%$, während lediglich in einigen osteuropäischen Staaten wie Litauen, Polen, Rumänien und Ungarn noch relativ hohe Preissteigerungsraten von mehr als $4 \%$ erreicht wurden. In Deutschland hat sich das Preisniveau bei einem minimalen Anstieg von 0,2 \% kaum verändert. Nach Einschätzung der Europäischen Kommission wird die Preisentwicklung im Jahr 2010 ebenfalls auf sehr niedrigem Niveau verbleiben und mit einem prognostizierten Anstieg von EU-weit 1,3\% nur leicht oberhalb des Vorjahres liegen.

Im Jahr 2009 nahm die Entwicklung der Arbeitsproduktivität im EU-Durchschnitt mit minus 2,1\% einen deutlich negativen Verlauf (Tabelle 2). Den stärksten Rückgang mussten wiederum die balti- 
Tabelle 2: Preise, Arbeitsproduktivität und Verteilungsspielraum in Europa 2007-2010

\begin{tabular}{|c|c|c|c|c|c|c|c|c|c|c|c|c|}
\hline & \multicolumn{4}{|c|}{ Preise } & \multicolumn{4}{|c|}{ Arbeitsproduktivität } & \multicolumn{4}{|c|}{ Verteilungsspielraum } \\
\hline & 2007 & 2008 & 2009 & 2010 & 2007 & 2008 & 2009 & 2010 & 2007 & 2008 & 2009 & 2010 \\
\hline \multicolumn{13}{|l|}{ Alte EU-Staaten } \\
\hline Belgien & 1,8 & 4,5 & 0,0 & 1,3 & 1,3 & $-0,8$ & $-2,1$ & 2,0 & 3,1 & 3,7 & $-2,1$ & 3,3 \\
\hline Dänemark & 1,7 & 3,6 & 1,1 & 1,5 & $-1,0$ & $-1,7$ & $-1,9$ & 3,7 & 0,7 & 1,9 & $-0,8$ & 5,2 \\
\hline Deutschland & 2,3 & 2,8 & 0,2 & 0,8 & 0,8 & $-0,1$ & $-4,9$ & 3,0 & 3,1 & 2,7 & $-4,7$ & 3,8 \\
\hline Finnland & 1,6 & 3,9 & 1,6 & 1,6 & 2,7 & $-0,3$ & $-4,1$ & 3,4 & 4,3 & 3,6 & $-2,5$ & 5,0 \\
\hline Frankreich & 1,6 & 3,2 & 0,1 & 1,1 & 1,0 & $-0,1$ & $-0,4$ & 2,1 & 2,6 & 3,1 & $-0,3$ & 3,2 \\
\hline Großbritannien & 2,3 & 3,6 & 2,0 & 1,4 & 1,9 & $-0,2$ & $-3,3$ & 1,8 & 4,2 & 3,4 & $-1,3$ & 3,2 \\
\hline Irland & 2,9 & 3,1 & $-1,7$ & $-0,6$ & 2,4 & $-2,2$ & 0,3 & 2,6 & 5,3 & 0,9 & $-1,4$ & 2,0 \\
\hline Italien & 2,0 & 3,5 & 0,8 & 1,8 & 0,3 & $-1,4$ & $-3,6$ & 1,5 & 2,3 & 2,1 & $-2,8$ & 3,3 \\
\hline Luxemburg & 2,7 & 4,1 & 0,0 & 1,8 & 2,0 & $-4,5$ & $-4,7$ & 2,4 & 4,7 & $-0,4$ & $-4,7$ & 4,2 \\
\hline Niederlande & 1,6 & 2,2 & 1,0 & 0,9 & 1,0 & 0,5 & $-4,4$ & 2,4 & 2,6 & 2,7 & $-3,4$ & 3,3 \\
\hline Österreich & 2,2 & 3,2 & 0,4 & 1,3 & 1,7 & 0,3 & $-2,9$ & 1,8 & 3,9 & 3,5 & $-2,5$ & 3,1 \\
\hline Portugal & 2,4 & 2,7 & $-0,9$ & 1,3 & 1,9 & $-0,4$ & $-0,6$ & 0,8 & 4,3 & 2,3 & $-1,5$ & 2,1 \\
\hline \multicolumn{13}{|l|}{ Neue EU-Staaten } \\
\hline Bulgarien & 7,6 & 12,0 & 2,5 & 2,3 & 3,3 & 2,7 & $-3,9$ & 0,3 & 10,9 & 14,7 & $-1,4$ & 2,6 \\
\hline Estland & 6,7 & 10,6 & 0,2 & 0,5 & 6,4 & $-3,7$ & $-5,1$ & 2,4 & 13,1 & 6,9 & $-4,9$ & 2,9 \\
\hline Lettland & 10,1 & 15,3 & 3,3 & $-3,7$ & 6,2 & $-5,2$ & $-6,9$ & 1,7 & 16,3 & 10,1 & $-3,6$ & $-2,0$ \\
\hline Litauen & 5,8 & 11,1 & 4,2 & $-0,7$ & 6,9 & 3,3 & $-10,8$ & $-1,5$ & 12,7 & 14,4 & $-6,6$ & $-2,2$ \\
\hline Malta & 0,7 & 4,7 & 1,8 & 2,0 & 0,8 & $-0,3$ & $-1,6$ & 0,4 & 1,5 & 4,4 & 0,2 & 2,4 \\
\hline Polen & 2,6 & 4,2 & 4,0 & 1,9 & 2,3 & 1,2 & 1,9 & 2,9 & 4,9 & 5,4 & 5,9 & 4,8 \\
\hline Rumänien & 4,9 & 7,9 & 5,6 & 3,5 & 5,9 & 7,6 & $-4,9$ & $-0,3$ & 10,8 & 15,5 & 0,7 & 3,2 \\
\hline Slowakei & 1,9 & 3,9 & 0,9 & 1,9 & 8,3 & 3,3 & $-3,9$ & 1,9 & 10,2 & 7,2 & $-3,0$ & 3,8 \\
\hline Slowenien & 3,8 & 5,5 & 0,9 & 1,7 & 3,7 & 0,7 & $-4,9$ & 3,4 & 7,5 & 6,2 & $-4,0$ & 5,1 \\
\hline Tschechien & 3,0 & 6,3 & 0,6 & 1,5 & 3,4 & 1,2 & $-2,8$ & 2,2 & 6,4 & 7,5 & $-2,2$ & 3,7 \\
\hline Ungarn & 7,9 & 6,0 & 4,0 & 4,0 & 1,1 & 1,9 & $-3,6$ & 0,3 & 9,0 & 7,9 & 0,4 & 4,3 \\
\hline Zypern & 2,2 & 4,4 & 0,2 & 3,1 & 1,8 & 0,8 & $-0,3$ & 0,2 & 4,0 & 5,2 & $-0,1$ & 3,3 \\
\hline Gesamte EU 27 & 2,4 & 3,7 & 1,0 & 1,3 & 1,1 & $-0,2$ & $-2,1$ & 2,0 & 3,5 & 3,5 & $-1,1$ & 3,3 \\
\hline Norwegen & 0,7 & 3,4 & 2,3 & 1,1 & $-1,3$ & $-1,3$ & $-1,1$ & 0,1 & $-0,6$ & 2,1 & 1,2 & 1,2 \\
\hline Quelle: Eurostat, Al & uropäisch & Kommiss & $n$, Berech & ngen des WSI. & & & & & & & AITTE & NGEN \\
\hline
\end{tabular}

schen Staaten hinnehmen, wo die Arbeitsproduktivität um minus 5,1\% in Estland, minus 6,9\% in Lettland und sogar minus $10,8 \%$ in Litauen zurückgegangen ist. In den alten EU-Staaten sank die Arbeitsproduktivität mit minus $4,9 \%$ am deutlichsten in Deutschland. Der starke Rückgang der Arbeitsproduktivität hat vor allem zwei Gründe: Zum einen ist er auf den Einbruch der Produktion zurückzuführen, der in den hochproduktiven Industriesektoren besonders ausgeprägt war. Zum andern hat in einigen Ländern die vorübergehende Arbeitszeitverkürzung zu einem deutlichen Rückgang der Produktivität je Arbeitnehmer geführt. Für 2010 erwartet die Europäische Kommission jedoch, dass sich die Entwicklung wieder deutlich erholen wird und die Arbeitsproduktivität im EU-Durchschnitt um 2,0 \% ansteigt.

Aufgrund der geringen Preissteigerung und der negativen Produktivitätsentwick- lung ergab sich im Jahr 2009 die historisch außergewöhnliche Situation, dass der aus der Gesamtsumme von Preis- und Produktivitätsentwicklung zusammengesetzte lohnpolitische Verteilungsspielraum mit minus 1,1\% im EU-Durchschnitt negativ ausfällt (Tabelle 2). Lediglich in sechs von 27 EU-Staaten sowie in Norwegen war 2009 der Verteilungsspielraum nach der klassischen Lohnformel noch positiv. In den alten EU-Staaten ging er mit minus $4,7 \%$ in Deutschland und Luxemburg am stärksten zurück. Innerhalb der neuen EU-Staaten wurde dieser Wert nur noch von Estland mit minus 4,9\% und Litauen mit minus 6,6 \% unterschritten. Für das Jahr 2010 prognostiziert die Europäische Kommission mit Ausnahme von Lettland und Litauen für alle anderen EU-Staaten wieder einen positiven Verteilungsspielraum, der im EU-Durchschnitt bei 3,3\% liegen soll. Folgt man der von vielen Öko- nomen ausgesprochenen Empfehlung, die Lohnpolitik nicht an der aktuellen Produktivitätsentwicklung, sondern an der mittelfristigen Trendproduktivität zu orientieren, so ergibt sich auch für 2009 im EU-Durchschnitt ein Verteilungsspielraum von $3 \%$ bis 3,5\%. Eine solche mittelfristige Orientierung hat in Krisenzeiten den Vorteil, dass die Lohnpolitik nicht prozyklisch agiert und durch mögliche Lohnkürzungen die Krise weiter verschärft.

\section{$\int_{\text {Die Entwicklung der }}$}

Die Analyse der Tariflohnentwicklung in Europa steht vor dem grundsätzlichen Problem, dass es bis heute keine offizielle europäische Tariflohnstatistik mit har- 


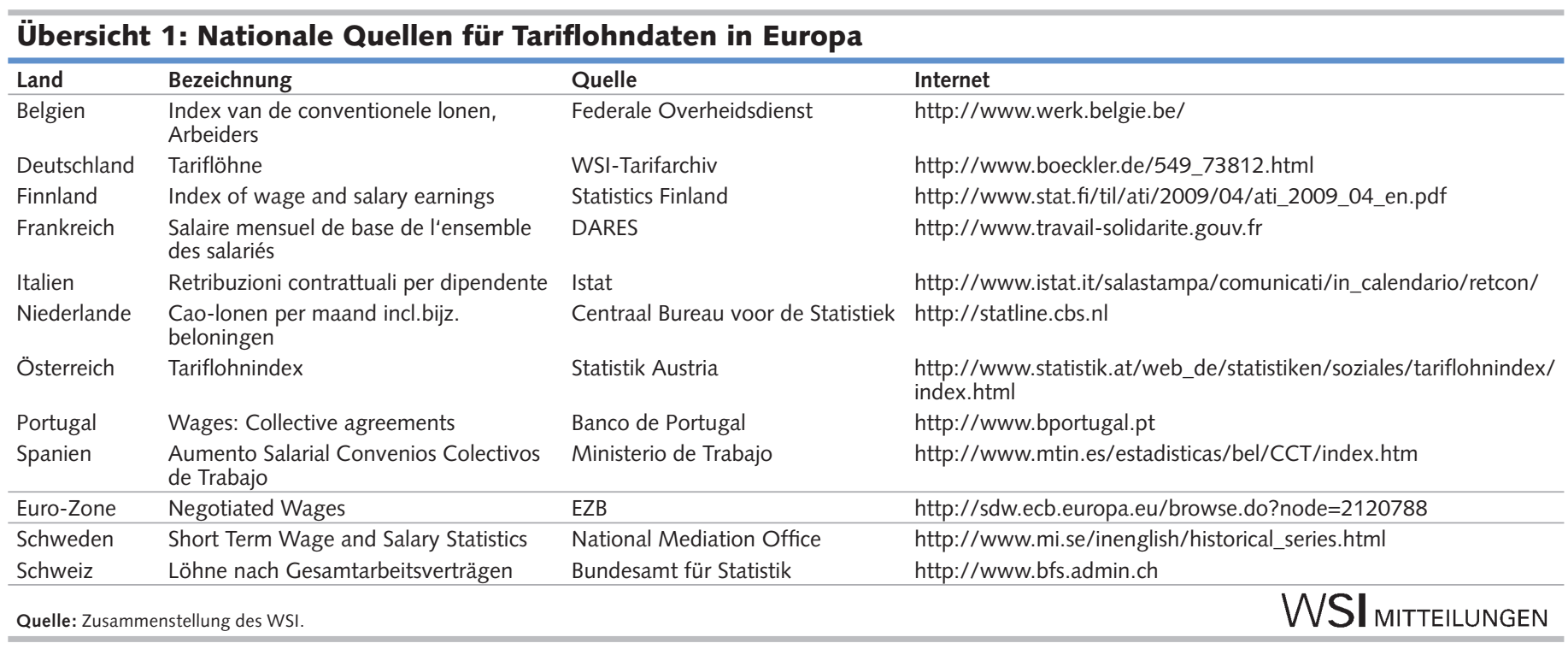

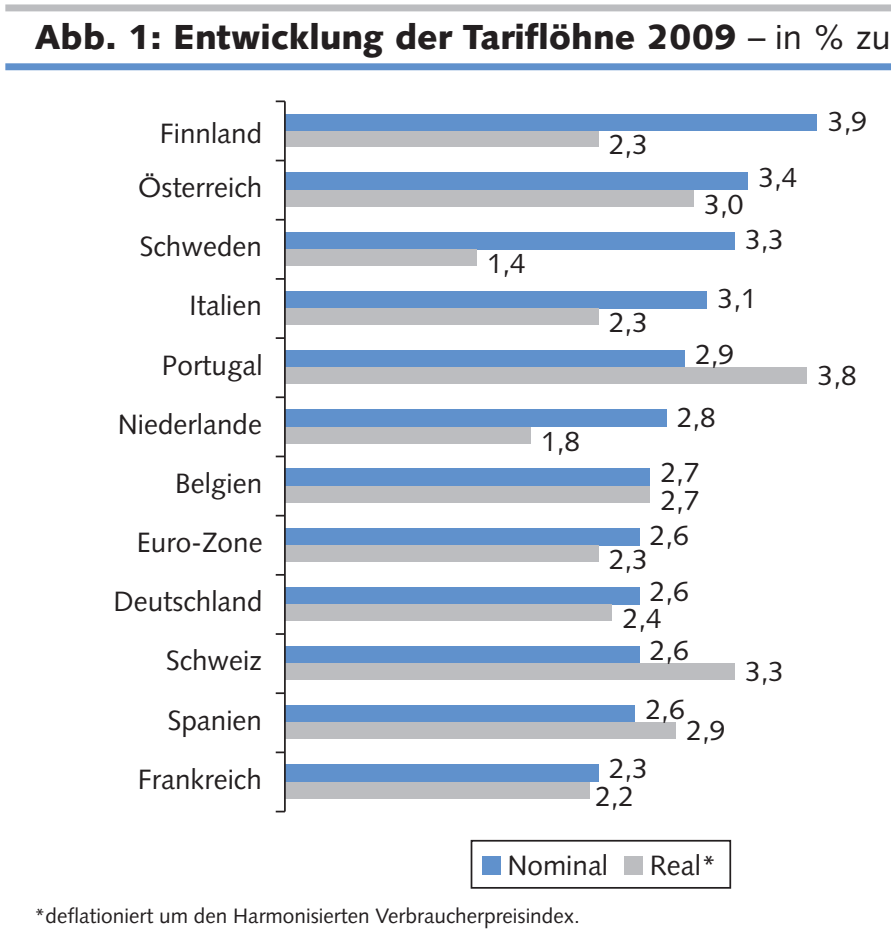

* deflationiert um den Harmonisierten Verbraucherpreisindex.

Quelle: EZB, Nationale Angaben; Berechnungen des WSI.

WSI MITTEILUNGEN

monisierten Tarifdaten gibt. ${ }^{2}$ Der einzige bislang zur Verfügung stehende offizielle Indikator ist der Tariflohnindex für die Euro-Zone, der von der Europäischen Zentralbank (EZB) berechnet wird. Die Grundlage hierfür bilden nicht-harmonisierte nationale Tarifdaten aus neun Mitgliedstaaten der Europäischen Währungsunion (EWU), die insgesamt etwa $95 \%$ des Euro-Raums abdecken. Hierzu gehören die großen EWU-Länder Deutschland, Frankreich, Italien und Spanien sowie Belgien, Finnland, Österreich, die Niederlande und Portugal, deren
Tarifdaten durch die Arbeitsministerien, die nationalen Statistikämter oder die nationalen Zentralbanken erhoben werden (Übersicht 1). Während die EZB lediglich den aggregierten Tariflohnindex für die gesamte Euro-Zone publiziert, werden in diesem Bericht erstmals auch die hierbei zugrunde liegenden nationalen Tarifdaten ausgewiesen. ${ }^{3}$ Darüber hinaus werden die vorliegenden Tariflohndaten für Schweden und die Schweiz in die Analyse einbezogen. Für die übrigen europäischen Länder - darunter alle Staaten aus Mittel und Osteuropa - liegen keine Tariflohnstatistiken vor.
Trotz Krise kam es im Jahr 2009 in vielen westeuropäischen Ländern zu relativ kräftigen Tariflohnsteigerungen. Die Spannbreite reichte von Finnland mit einer nominalen Tariflohnerhöhung von 3,9 \% bis zu Frankreich mit 2,3\% (Abbildung 1). Für die gesamte Euro-Zone weist der Tariflohnindex der EZB 2009 einen nominalen Zuwachs von 2,6\% aus. Dieser lag zwar unterhalb der Steigerungsrate von 3,2 \% des Boomjahrs 2008, aber oberhalb der Vorjahre 2007 $(2,1 \%)$ und 2006 (2,3\%). Deutschland be-

2 Seit einigen Jahren veröffentlicht das European Industrial Relations Observatory (EIRO) regelmäßig Daten über die Entwicklung der Tariflöhne in Europa (vgl. zuletzt Carley 2009a). Eine Überprüfung der vom EIRO verwendeten nationalen Ursprungsdaten macht jedoch deutlich, dass diese große methodische Mängel aufweisen und nicht miteinander vergleichbar sind. Zum einen werden in den EIRO-Daten mitunter sehr unterschiedliche Bereiche und Zeiträume erfasst. Zum anderen werden aufgrund fehlender nationaler Tariflohnstatistiken oft Datenquellen verwendet, die faktisch keine Tarif-, sondern Effektivlohnentwicklungen darstellen. Qualitative Berichte über die Tarifauseinandersetzungen in den einzelnen EU-Staaten finden sich in den laufenden nationalen Beiträgen zum EIRO (http://www. eurofound.europa.eu/eiro) sowie in dem gemeinsam vom Europäischen Gewerkschaftsinstitut und dem Amsterdam Institute for Advanced Labour Studies (AIAS) herausgegebenen Collective Bargaining Newsletter (http://www.etui-rehs.org/ research/Publications/Newsletters/Collectivebargaining-newsletter).

3 Für Deutschland werden von der EZB die Daten des Tariflohnindex der Deutschen Bundesbank verwendet. Im Gegensatz dazu werden hier die Daten des WSI-Tarifarchivs benutzt, die in der Regel etwas höher ausfallen. Dies liegt vor allem daran, dass bei dem Bundesbankindex auch Branchen berücksichtig werden, in denen ein Tarifvertrag ausgelaufen ist, ohne dass im Berichtsjahr ein neuer Tarifabschluss getätigt wurde. 
fand sich mit einer nominalen Tariflohnerhöhung von 2,6 \% im europäischen Durchschnitt (Bispinck/WSI-Tarifarchiv 2010).

Aufgrund der geringen und in einigen Ländern sogar negativen Preisentwicklung kam es in allen hier berücksichtigten Ländern zu einer deutlichen Erhöhung der realen Tariflöhne. Den höchsten Reallohnzuwachs verzeichnete dabei Portugal mit 3,8 \%, gefolgt von der Schweiz mit 3,3\% und Österreich mit 3,0\%. Die geringsten Reallohnsteigerungen gab es hingegen mit $1,4 \%$ in Schweden und 1,8 \% in den Niederlanden. Deutschland bewegte sich mit einem tariflichen Reallohnzuwachs von 2,4 \% im europäischen Mittelfeld.

Angesichts der negativen Entwicklung der Arbeitsproduktivität lagen die Tarifabschlüsse in allen Ländern deutlich über dem auf das Jahr 2009 bezogenen Verteilungsspielraum und führten erstmals seit Langem wieder zu einem Anstieg der Lohnquote. Das ist für Krisenzeiten typisch, da die Lohnentwicklung immer mit einer gewissen Zeitverzögerung auf die allgemeine Konjunkturentwicklung reagiert. So wurden z. B. viele Tarifabschlüsse für 2009 bereits in den Boomjahren 2007 und 2008 getätigt. Im Laufe des Jahres 2009 sind die vereinbarten Tariferhöhungen in den meisten Ländern jedoch immer geringer geworden. Während der Tariflohnindex der EZB im ersten Quartal 2009 noch um 3,2 \% über dem entsprechenden Wert der Vorperiode lag, waren es im vierten Quartal 2009 nur noch 2,1 \%. Für 2010 ist demnach mit deutlich geringeren Tariflohnerhöhungen zu rechnen.

Über das gesamte letzte Jahrzehnt hinweg zeigt die Entwicklung der Tariflöhne in Europa sehr große Unterschiede ( $A b$ bildung 2). Während zwischen 2000 und 2009 die nominalen Tariflöhne in der Schweiz um 19,6\% anstiegen, wuchsen sie in Finnland mit 41,6 \% mehr als doppelt so schnell. In Deutschland fiel der nominale Tariflohnanstieg mit $22 \%$ nach der Schweiz am geringsten aus. Ähnlich große Unterschiede ergeben sich nach Abzug der Preissteigerungsrate bei den realen Tariflohnzuwächsen. Diese waren in Finnland mit 22,1 \% am größten, gefolgt von Schweden mit 16,3\% und der Schweiz, die trotz relativ geringer Nominallohnerhöhungen mit $11 \%$ ein relativ hohes Reallohnwachstum verzeichnete. In Deutschland stiegen die Tariflöhne real um 5,1\%. In den Niederlanden war der tarifliche Reallohnzuwachs mit 2,6\% am geringsten. ${ }^{4}$
Abb. 2: Entwicklung der Tariflöhne 2000-2009 - in \% -

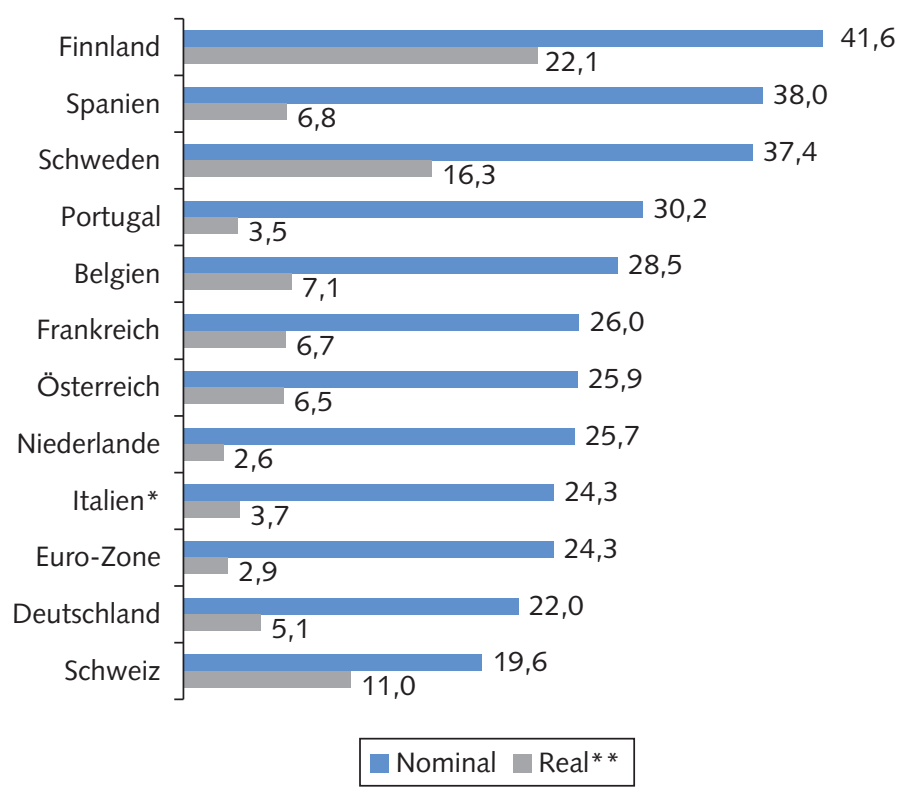

*2001-2009; ** deflationiert um den Harmonisierten Verbraucherpreisindex.

Quelle: EZB, Nationale Angaben; Berechnungen des WSI.

WSI MITTEILUNGEN

\section{3 \\ Die Entwicklung der Effektivlöhne}

Im Gegensatz zu den Tariflöhnen, die auf tarifvertraglichen Vereinbarungen beruhen, handelt es sich bei den Effektivlöhnen um die in der Volkswirtschaftlichen Gesamtrechnung (VGR) ausgewiesenen Löhne, die tatsächlich an alle Beschäftigten gezahlt wurden. Die oft zu beobachtenden Unterschiede zwischen Tarif- und Effektivlohnentwicklung werden als Lohndrift bezeichnet und können verschiedene Ursachen haben. Inwieweit die Tariflohnerhöhungen tatsächlich auch effektiv beim Durchschnitt aller Beschäftigten ankommen, hängt vor allem von der Tarifbindung ab, die in Europa zwischen mehr als $90 \%$ in einigen westeuropäischen Ländern und weniger als $20 \%$ in den baltischen Staaten variiert (Schulten 2010). Darüber hinaus besteht in einigen Ländern die Möglichkeit, unter bestimmten Bedingungen auf betrieblicher Ebene von sektoralen Tariferhöhungen nach unten hin abzuweichen. Umgekehrt werden in vielen Ländern von den Unternehmen nach wie vor übertarifliche Leistungen bezahlt. Schließlich wird das Verhältnis von Tarif- und Effektivlöhnen auch durch die Entwicklung der effektiven
Arbeitszeit beeinflusst, wobei z.B. Überstunden eine positive, Kurzarbeit hingegen eine negative Lohndrift befördern. Im Folgenden wird die Entwicklung der Effektivlöhne auf der Basis der AMECO-Datenbank der Europäischen Kommission analysiert. ${ }^{5}$

Im Jahr 2009 stiegen die Nominallöhne im EU-Durchschnitt um 1,7 \% und damit deutlich geringer als noch in den Vorjahren, wo sie um 3,3 \% (2008) bzw. 3,2 \% (2007) zunahmen (Tabelle 3). Für 2010 prognostiziert die Europäische Kommission mit 1,5\% ein ähnlich niedriges Nominallohnwachstum wie 2009. Deutlich ausgeprägter als bei den Tariflöhnen wirkt sich damit die Krise bereits stark dämpfend auf die Entwicklung der Effektivlöhne aus. Im Jahr 2009 dürfte demnach die Lohndrift zwischen Tariflohn- und Effektivlohnentwicklung in vielen europäischen Ländern negativ sein. Dies liegt neben betrieblichen Lohnkürzungen vor allem auch an den

4 Der für den Zeitraum 2000 bis 2009 ungewöhnlich niedrige reale Zuwachs des EZB-Tarifindex dürfte damit zusammenhängen, dass er für Deutschland auf der Basis der Tarifdaten der Bundesbank deutlich geringere Werte enthält. Vgl. Fußnote 3.

5 Streng genommen werden in der AMECO-Datenbank der Europäischen Kommission keine "Löhne", sondern "Arbeitnehmerentgelte" (definiert als Bruttolöhne plus Arbeitgeberanteile zur Sozialversicherung) ausgewiesen. Der hier im Folgenden verwendete weite Lohnbegriff umfasst demnach die gesamten Arbeitskosten. 
Tabelle 3: Lohnentwicklung und Verteilungsbilanz in Europa 2007-2010

\begin{tabular}{|c|c|c|c|c|c|c|c|c|c|c|c|c|}
\hline & \multicolumn{4}{|c|}{ Nominallöhne } & \multicolumn{4}{|c|}{ Reallöhne } & \multicolumn{4}{|c|}{ Verteilungsbilanz } \\
\hline & 2007 & 2008 & 2009 & 2010 & 2007 & 2008 & 2009 & 2010 & 2007 & 2008 & 2009 & 2010 \\
\hline \multicolumn{13}{|l|}{ Alte EU-Staaten } \\
\hline Belgien & 3,5 & 3,0 & 0,7 & 1,7 & 1,7 & $-1,5$ & 0,7 & 0,5 & 0,4 & $-0,7$ & 2,8 & $-1,5$ \\
\hline Dänemark & 3,1 & 4,9 & 3,4 & 3,0 & 1,4 & 1,3 & 2,3 & 1,4 & 2,4 & 3,0 & 4,2 & $-2,3$ \\
\hline Deutschland & 1,0 & 2,1 & 0,0 & 1,3 & $-1,3$ & $-0,7$ & $-0,2$ & 0,5 & $-2,1$ & $-0,6$ & 4,7 & $-2,5$ \\
\hline Finnland & 3,4 & 5,3 & 3,4 & 2,7 & 1,8 & 1,4 & 1,8 & 1,2 & $-0,9$ & 1,7 & 5,9 & $-2,2$ \\
\hline Frankreich & 2,5 & 2,7 & 1,2 & 1,5 & 0,9 & $-0,5$ & 1,1 & 0,4 & $-0,1$ & $-0,4$ & 1,5 & $-1,7$ \\
\hline Großbritannien & 4,9 & 2,3 & 1,2 & 1,2 & 2,6 & $-1,3$ & $-0,8$ & $-0,2$ & 0,7 & $-1,1$ & 2,5 & $-2,0$ \\
\hline Irland & 6,0 & 5,5 & $-1,6$ & $-1,8$ & 3,1 & 2,4 & 0,1 & $-1,2$ & 0,7 & 4,6 & $-0,2$ & $-3,8$ \\
\hline Italien & 2,2 & 3,3 & 1,7 & 1,6 & 0,2 & $-0,2$ & 0,9 & $-0,2$ & $-0,1$ & 1,2 & 4,5 & $-1,7$ \\
\hline Luxemburg & 3,6 & 2,0 & 1,2 & 1,8 & 0,9 & $-2,1$ & 1,2 & $-0,1$ & $-1,1$ & 2,4 & 5,9 & $-2,5$ \\
\hline Niederlande & 3,4 & 3,8 & 2,6 & 2,5 & 1,8 & 1,6 & 1,6 & 1,6 & 0,8 & 1,1 & 6,0 & $-0,8$ \\
\hline Österreich & 3,0 & 3,1 & 3,0 & 2,0 & 0,8 & $-0,1$ & 2,6 & 0,7 & $-0,9$ & $-0,4$ & 5,5 & $-1,1$ \\
\hline Portugal & 3,4 & 3,1 & 4,7 & 2,0 & 1,0 & 0,4 & 5,6 & 0,6 & $-0,9$ & 0,8 & 6,2 & $-0,2$ \\
\hline Schweden & 5,1 & 1,6 & 2,3 & 2,1 & 3,4 & $-1,7$ & 0,4 & 0,4 & 3,0 & $-0,6$ & 2,8 & $-2,8$ \\
\hline \multicolumn{13}{|l|}{ Neue EU-Staaten } \\
\hline Bulgarien & 17,9 & 19,3 & 7,8 & 2,5 & 10,3 & 7,3 & 5,3 & 0,2 & 7,0 & 4,6 & 9,2 & $-0,1$ \\
\hline Estland & 24,8 & 10,1 & $-4,5$ & $-2,7$ & 18,1 & $-0,5$ & $-4,7$ & $-3,2$ & 11,7 & 3,2 & 0,4 & $-5,6$ \\
\hline Lettland & 34,8 & 16,7 & $-12,0$ & $-8,0$ & 24,7 & 1,4 & $-15,3$ & $-4,3$ & 18,5 & 6,6 & $-8,4$ & $-6,0$ \\
\hline Litauen & 13,9 & 12,9 & $-6,8$ & $-5,7$ & 8,1 & 1,8 & $-11,0$ & $-5,0$ & 1,2 & $-1,5$ & $-0,2$ & $-3,5$ \\
\hline Malta & 1,5 & 3,4 & 2,5 & 2,1 & 0,8 & $-1,3$ & 0,7 & 0,1 & 0,0 & $-1,0$ & 2,3 & $-0,3$ \\
\hline Polen & 4,9 & 8,1 & 4,5 & 1,5 & 2,3 & 3,9 & 0,5 & $-0,4$ & 0,0 & 2,7 & $-1,4$ & $-3,3$ \\
\hline Rumänien & 22,0 & 21,7 & 4,8 & 5,5 & 17,1 & 13,8 & $-0,8$ & 1,9 & 11,2 & 6,2 & 4,1 & 2,2 \\
\hline Slowakei & 8,8 & 8,7 & 3,6 & 3,9 & 6,9 & 4,8 & 2,7 & 2,0 & $-1,4$ & 1,5 & 6,6 & 0,1 \\
\hline Slowenien & 6,5 & 6,7 & 2,9 & 1,7 & 2,7 & 1,2 & 2,0 & 0,0 & $-1,0$ & 0,5 & 6,9 & $-3,4$ \\
\hline Tschechien & 6,4 & 6,0 & 0,5 & 1,6 & 3,4 & $-0,3$ & $-0,1$ & 0,1 & 0,0 & $-1,5$ & 2,7 & $-2,1$ \\
\hline Ungarn & 6,8 & 6,6 & $-1,4$ & 0,0 & $-1,1$ & 0,6 & $-5,4$ & $-4,1$ & $-2,2$ & $-1,3$ & $-1,8$ & $-4,4$ \\
\hline Zypern & 2,1 & 4,0 & 2,0 & 1,8 & $-0,1$ & $-0,4$ & 1,8 & $-1,3$ & $-1,9$ & $-1,2$ & 2,1 & $-1,5$ \\
\hline Gesamte EU 27 & 3,2 & 3,3 & 1,7 & 1,5 & 0,8 & $-0,4$ & 0,7 & 0,2 & $-0,3$ & $-0,2$ & 2,8 & $-1,8$ \\
\hline Norwegen & 5,7 & 5,9 & 3,2 & 2,8 & 5,0 & 2,5 & 0,9 & 1,7 & 6,3 & 3,8 & 2,0 & 1,6 \\
\hline Quelle: Eurostat; Al & Europäisc & en Kommi & sion; Statis & sches Bur & für Deuts & land); Ber & echnungen & s WSI. & & & IITTEI & GEN \\
\hline
\end{tabular}

Maßnahmen zur Beschäftigungssicherung durch vorübergehende Arbeitszeitverkürzung, die sich negativ auf die Effektivlöhne je Beschäftigten auswirken.

In fünf EU-Staaten kam es 2009 erstmals zu einem Rückgang der Nominallöhne. Am stärksten hiervon betroffen waren wiederum die baltischen Staaten, die Nominallohnkürzungen von - im Falle Lettlands - bis zu $12 \%$ hinnehmen mussten. Ebenfalls negativ war die Nominallohnentwicklung mit minus $1,6 \%$ in Irland und minus $1,4 \%$ in Ungarn. Während in Deutschland die effektiven Nominallöhne stagnierten, stiegen sie in den meisten EU-Staaten zwischen $1 \%$ und $3 \%$. Die höchsten Nominallohnzuwächse gab es in Rumänien (4,8 \%), Portugal (4,7 \%) und Polen (4,5 \%). Für 2010 geht die Europäische Kommission davon aus, dass die Nominallöhne in der Regel zwischen $1 \%$ und $2 \%$ zunehmen. Für die baltischen Staaten und Irland werden hingegen weitere Nominallohnkürzungen vorhergesagt.

Angesichts der geringen Preisentwicklung kam es 2009 trotz geringer Nominallohnzuwächse im EU-Durchschnitt zu einem leichten Anstieg der Reallöhne um $0,7 \%$. Im Gegensatz dazu waren sie 2008 um $0,4 \%$ zurückgegangen, was an den damals relativ hohen Inflationsraten lag. Für 2010 erwartet die Europäische Kommission, dass die Reallöhne mit 0,2 \% de facto stagnieren, wobei für zehn von 27 EU-Staaten ein Reallohnrückgang prognostiziert wird.

Die Verteilungsbilanz, die sich aus dem Saldo aus Nominallohnentwicklung und dem Verteilungsspielraum ergibt, ist 2009 in den meisten EU-Staaten deutlich positiv. Im EU-Durchschnitt lagen die Nominalzuwächse um 2,8 Prozentpunkte über der Preis- und Produktivitätsentwicklung. Allerdings gab es 2009 immer noch fünf
EU-Staaten, die trotz Krise eine negative Verteilungsbilanz verzeichneten. Nach den Erwartungen der Europäischen Kommission wird sich der Verteilungstrend jedoch bereits 2010 wieder umkehren. Mit Ausnahme von Rumänien und der Slowakei (und außerhalb der EU Norwegen) wird allen EU-Staaten eine zum Teil deutlich negative Verteilungsbilanz vorausgesagt, die im EU-Durchschnitt um 1,8 Prozentpunkte unterhalb des Verteilungsspielraums liegen soll. Damit käme es europaweit wieder zu einer äußerst restriktiven Lohnentwicklung.

\section{1 \\ Arbeitszeiten in Europa}

Bei den Arbeitszeiten liegen bislang europaweit vergleichende Daten nur bis 2008 
vor, sodass der Einfluss der Krise noch nicht genau bestimmt werden kann. Angesichts zahlreicher staatlicher Förderprogramme zur Beschäftigungssicherung durch befristete Arbeitszeitverkürzung dürften 2009 die effektiven Arbeitszeiten in einigen europäischen Ländern zurückgegangen sein (European Foundation 2009). Auch wenn eine generelle Arbeitszeitverkürzung in keinem europäischen Land auf der Tagesordnung stand, so haben sich die Tarifvertragsparteien in einigen Ländern darum bemüht, die Spielräume für eine zeitweise Reduzierung der Arbeitszeit zu erweitern (Glassner/Keune 2010).

Im Jahr 2008 lag die tarifvertraglich vereinbarte Wochenarbeitszeit im EUDurchschnitt unverändert bei 38,6 Stunden (Tabelle 4). In zehn EU-Staaten, zu denen neben Griechenland und Malta vor allem Länder aus Mittel- und Osteuropa gehören, betrug die vereinbarte Standardwochenarbeitszeit 40 Stunden. In acht Ländern lag die Wochenarbeitszeit zwischen 38 und 39 Stunden und in neun Ländern unterhalb von 38 Stunden. Deutschland bewegte sich mit 37,6 Wochenarbeitsstunden im unteren Mittelfeld. Die kürzeste tarifvertragliche Wochenarbeitszeit bestand hingegen in Frankreich, wo seit Ende der 1990er Jahre die 35-Stunden-Woche als gesetzlich festgeschriebene Regelarbeitszeit gilt.

Die effektiven Wochenarbeitszeiten von Vollzeitbeschäftigten lagen 2008 noch nahezu überall in Europa über den tarifvertraglichen Normen. Im EU-Durchschnitt arbeitete ein vollzeitbeschäftigter Arbeitnehmer 40,5 Stunden pro Woche und damit 1,9 Stunden mehr als im Tarifvertrag vereinbart worden ist. Die längsten effektiven Arbeitszeiten fanden sich mit 42,4 Stunden in Großbritannien und 42,2 Stunden in Österreich. In 17 von 27 EU-Staaten lag die effektive Wochenarbeitszeit bei 40 und mehr Stunden. Deutschland befand sich mit durchschnittlich 40,4 Stunden pro Woche im EU-Durchschnitt. Die kürzesten effektiven Arbeitszeiten mit einer Dauer von unter 39 Stunden pro Woche existieren in Irland und den Niederlanden. Gegenüber dem Vorjahr sind auch die effektiven Arbeitszeiten weitgehend konstant geblieben.
Tabelle 4: Tarifliche und effektive Wochenarbeitszeiten in der Europäischen Union 2008

\begin{tabular}{|c|c|c|c|c|c|}
\hline Alte EU-Staaten & Tarifvertraglich* & Effektiv* * & Neue EU-Staaten & Tarifvertraglich* & Effektiv** \\
\hline Belgien & 37,6 & 39,1 & Bulgarien & 40,0 & 41,5 \\
\hline Dänemark & 37,0 & 39,3 & Estland & 40,0 & 40,7 \\
\hline Deutschland & 37,6 & 40,4 & Lettland & 40,0 & 41,1 \\
\hline Finnland & 37,5 & 39,1 & Litauen & 40,0 & 39,9 \\
\hline Frankreich & 35,0 & 39,3 & Malta & 40,0 & 40,4 \\
\hline Griechenland & 40,0 & 40,8 & Polen & 40,0 & 41,2 \\
\hline Großbritannien & 37,3 & 42,4 & Rumänien & 40,0 & 41,3 \\
\hline Irland & 39,0 & 38,8 & Slowakei & 38,4 & 40,8 \\
\hline Italien & 38,0 & 39,2 & Slowenien & 40,0 & 41,5 \\
\hline Luxemburg & 39,0 & 39,8 & Tschechien & 38,0 & 41,3 \\
\hline Niederlande & 37,5 & 38,9 & Ungarn & 40,0 & 40,6 \\
\hline Österreich & 38,8 & 42,2 & Zypern & 38,0 & 40,6 \\
\hline Portugal & 38,2 & 40,2 & & & \\
\hline Schweden & 37,5 & 39,9 & & & \\
\hline Spanien & 38,3 & 40,6 & & & \\
\hline \multicolumn{4}{|c|}{ EU 27} & 38,6 & 40,5 \\
\hline \multicolumn{6}{|c|}{$\begin{array}{l}\text { * Durchschnittliche tarifvertraglich vereinbarte Wochenarbeitszeit. } \\
\text { ** Durchschnittliche effektive Arbeitszeit von Vollzeitbeschäftigten. }\end{array}$} \\
\hline
\end{tabular}

Abb. 3: Entwicklung der preislichen Wettbewerbsfähigkeit auf Basis der Lohnstückkostenentwicklung 1999-2009* - in \% -

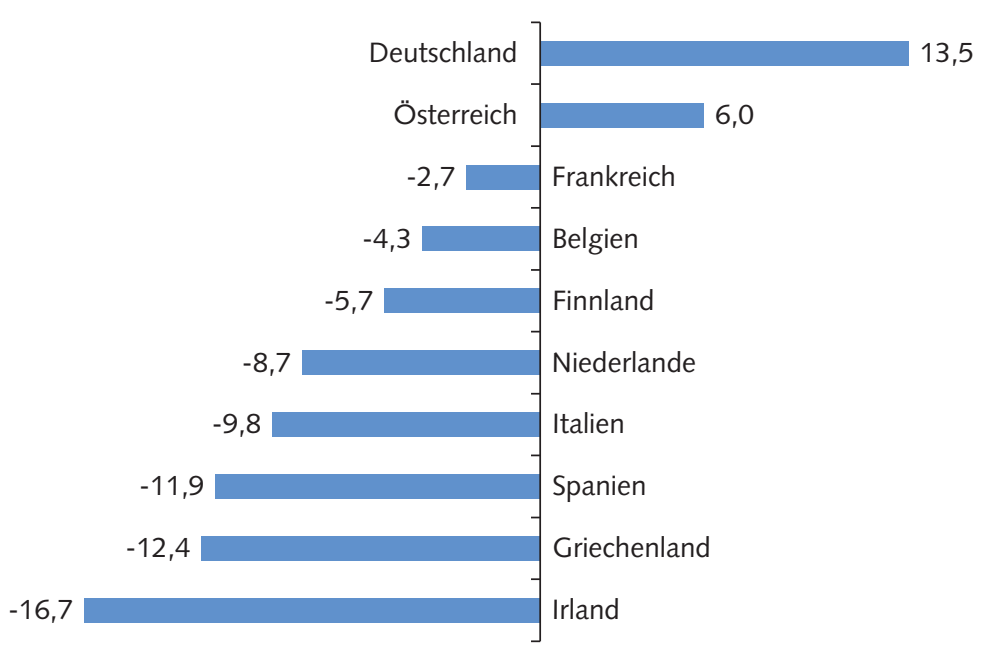

*Entwicklung des harmonisierten Indikators der Wettbewerbsfähigkeit; Berechnung für 2009 auf Grundlage der ersten drei Quartale.

Quelle: EZB, Nationale Angaben; Berechnungen des WSI.

WSI MITTELLUNGEN

\section{5 \\ Ausblick: Lohnpolitik in der Krise}

In Krisenzeiten geraten die Löhne gewöhnlich besonders stark unter Druck. Während Unternehmen ihr Heil in weiteren Kostensenkungen suchen, sind die Beschäftigten umso mehr bereit, auf Lohnerhöhungen zu verzichten, wenn sie ihren Arbeitsplatz in Gefahr sehen. Wenn auch mit einiger Zeitverzögerung, so hat die Lohnentwicklung im Laufe des Jahre 2009 deutlich an Dyna- mik verloren. Im Jahr 2010 wird nach den Prognosen der Europäischen Kommission der Lohnzuwachs noch schwächer ausfallen und zumindest die Reallöhne werden weitgehend stagnieren. Hierzu passt, dass in vielen nationalen Tarifauseinandersetzungen die Arbeitgeber mittlerweile eine Null-Runde bei den Löhnen fordern oder sogar für Lohnkürzungen eintreten. Vor dem Hintergrund der in der Krise stark angestiegenen Staatsverschuldung gehen auch die öffentlichen Arbeitgeber vermehrt dazu über, die Löhne im öffentlichen Dienst einzufrieren. In einigen Ländern - wie das aktuelle Beispiel Griechenland 
zeigt - werden die Regierungen auch von internationalen Organisationen wie der Europäischen Union und dem Internationalen Währungsfond dazu gedrängt, die Löhne ihrer Staatsdiener zu kürzen.

Die 2010 zu erwartende Lohnentwicklung ist umso problematischer, als die Löhne sich im letzten Jahrzehnt innerhalb Europas immer weiter auseinanderentwickelt haben. Während insbesondere Deutschland durch eine stark restriktive Lohnentwicklung seine preisliche Wettbewerbfähigkeit gegenüber den europäischen Nachbarn immer mehr verbessert hat, mussten andere Länder durch höhere Lohnabschlüsse Wettbewerbsverluste hinnehmen (Abbildung 3). Das Ergebnis war eine zunehmende Ungleichheit von Ländern mit wachsenden Exportüberschüssen und Ländern, die diese Exporte durch zunehmende Verschuldung finanziert haben.
Wollten Länder wie Griechenland, Irland und Spanien heute in ihrer preislichen Wettbewerbfähigkeit mit Deutschland gleichziehen, so müssten sie theoretisch ihre Löhne auf einen Schlag um mehr als $20 \%$ kürzen. Die aktuellen Tarifabschlüsse in Deutschland setzen diese Länder zusätzlich unter Druck (Rohan 2010; Flassbeck 2010).

Im letzten Jahrzehnt hat sich in Europa ein lohnpolitisches Entwicklungsmuster etabliert, das zu einer zentralen Ursache der gegenwärtigen Krise geworden ist. Es hat einerseits dazu beigetragen, dass sich die außenwirtschaftlichen Ungleichgewichte zwischen den europäischen Staaten immer weiter vergrößert haben. Andererseits hat es dazu geführt, dass die private Konsumnachfrage insbesondere in Deutschland systematisch unterentwickelt blieb und damit die Wachstumspotenziale in ganz
Europa nicht ausgeschöpft wurden (Joebkes et al. 2009). In der aktuellen Krise droht sich dieses lohnpolitische Entwicklungsmuster nun noch einmal zu verschärfen.

Die Alternative läge darin, die Bemühungen für eine europäische Koordinierung der Lohnpolitik deutlich zu verstärken. Die vom Europäischen Gewerkschaftsbund (EGB 2009) für die Tarifrunde 2010 herausgegebene Parole „No wage freeze and wage cuts" markiert dabei nur die unterste Haltelinie, um einen europaweiten Lohnsenkungswettlauf und den damit verbundenen Deflationsgefahren zu entgegnen. Notwendig ist darüber hinaus eine grundlegende Diskussion darüber, wie nach der Krise ein alternatives ökonomisches Entwicklungsmodell in Europa aussehen und welche Bedeutung hierbei der Lohnpolitik zukommen soll.

\section{LITERATUR}

Bispinck, R./WSI-Tarifarchiv (2010): Tarifpolitischer Jahresbericht 2009: Tarifverdienste mit kräftigem Plus - Effektivverdienste im Minus, in: WSI-Mitteilungen 3, S. 143-151

Carley, M. (2009a): Pay Developments - 2008, EIROnline http://www. eurofound.europa.eu/docs/eiro/tn0904029s/tn0904029s.pdf Carley, M. (2009b): Working Time Developments - 2008, EIROnline, www.eurofound.europa.eu/docs/eiro/tn0903039s/tn0903039s.pdf Europäische Kommission (2009): European Economic Forecast Autum 2009, European Economy 10 Europäische Kommission (2010a): Fragile Recovery in Progress in the EU, EU Interim Forecast, Februar

Europäische Kommission (2010b): Quarterly EU Labour Market Review, Winter

Europäischer Gewerkschaftsbund (EGB) (2009): Guidelines for the Coordination of Collective Bargaining, angenommene Resolution des EGB Exekutivausschusses, am 1.-2. Dezember 2009 (www.etuc.org/ IMG/pdf_01-02-2009Resolution-EN-No-to-wages-freezes-and-wagecuts.pdf)

European Foundation for the Improvement of Living and Working Conditions (European Foundation) (2009): Tackling the Recession: Employment related Public Initiatives in the EU Member States and Norway, Dublin
Flassbeck, H. (2010): Putting Employment Security first will diminish Demand - a Warning from Germany, Global Labor Column Nr. 13 (www.global-labour-university.org/fileadmin/GLU_Column/papers/ no_13_flassbeck.pdf)

Glassner, V./Keune, M. (2010): Collective Bargaining Responses to the Economic Crisis in Europe, ETUI Policy Brief 1

Joebkes, H./Schmalzbauer, A./Zwiener, R. (2009): Der Preis für den Exportweltmeister Deutschland: Reallohnrückgang und geringes Wirtschaftswachstum, IMK Studies 4, August

Massarelli, N. (2009): European Union Labour Force Survey-Annual Results, in: Eurostat (Hrsg.): Data in Focus 33

Rohan, B. (2010): German Wage Deal widens Competitive Gulf within EMU, Reuters News, 26. Februar

Schulten, T. (2009): Zehn Jahre europäische Koordinierung der Tarifpolitik - eine Zwischenbilanz, in: Schlatermund, H./Flore, M. (Hrsg.): Zukunft von Arbeitsbeziehungen und Arbeit in Europa (ZAUBER),

Osnabrück, S. 103-118

Schulten, T. (2010): Das deutsche Tarifvertragssystem im europäischen Vergleich, in: Bispinck, R./Schulten, T. (Hrsg.): Zukunft der Tarifautonomie, Hamburg, S. 193-204 